\title{
OPEN Abiraterone induces SLCO1B3 expression in prostate cancer via microRNA-579-3p
}

\author{
Roberto H. Barbier ${ }^{1}$, Edel M. McCrea ${ }^{1}$, KristiY. Lee ${ }^{1}$, Jonathan D. Strope ${ }^{1}$, Emily N. Risdon ${ }^{1}$, \\ Douglas K. Price ${ }^{1}$, Cindy H. Chau ${ }^{1}$ \& William D. Figg ${ }^{1,2}$
}

Understanding mechanisms of resistance to abiraterone, one of the primary drugs approved for the treatment of castration resistant prostate cancer, remains a priority. The organic anion polypeptide 1B3 (OATP1B3, encoded by SLCO1B3) transporter has been shown to transport androgens into prostate cancer cells. In this study we observed and investigated the mechanism of induction of SLCO1B3 by abiraterone. Prostate cancer cells (22Rv1, LNCaP, and VCAP) were treated with antiandrogens and assessed for $S L C O 1 B 3$ expression by $q P C R$ analysis. Abiraterone treatment increased SLCO1B3 expression in 22Rv1 cells in vitro and in the 22Rv1 xenograft model in vivo. MicroRNA profiling of abiraterone-treated 22Rv1 cells was performed using a NanoString nCounter miRNA panel followed by miRNA target prediction. TargetScan and miRanda prediction tools identified hsa-miR579-3p as binding to the $3^{\prime}$-untranslated region ( $3^{\prime} U T R$ ) of the SLCO1B3. Using dual luciferase reporter assays, we verified that hsa-miR-579-3p indeed binds to the SLCO1B3 3'UTR and significantly inhibited SLCO1B3 reporter activity. Treatment with abiraterone significantly downregulated hsa-miR-579-3p, indicating its potential role in upregulating $S L C O 1 B 3$ expression. In this study, we demonstrated a novel miRNA-mediated mechanism of abiraterone-induced SLCO1B3 expression, a transporter that is also responsible for driving androgen deprivation therapy resistance. Understanding mechanisms of abiraterone resistance mediated via differential miRNA expression will assist in the identification of potential miRNA biomarkers of treatment resistance and the development of future therapeutics.

Androgens and androgen receptor (AR) signaling drive prostate carcinogenesis. While androgen ablation therapy (ADT) has been the cornerstone of treatment for advanced prostate cancer, patients inevitably progress to castrate-resistant prostate cancer (CRPC) despite castrate levels of androgens (serum testosterone $<50 \mathrm{ng} / \mathrm{mL}$ ). The current treatment armamentarium includes androgen biosynthesis inhibitors (e.g., abiraterone) and AR antagonists (e.g., enzalutamide, apalutamide, and darolutamide) that target persistent androgen production and AR signaling. Although these drugs prolong survival in men with CRPC, resistance eventually ensues; therefore, understanding mechanisms of resistance will facilitate future drug discovery and development.

Multiple groups have demonstrated SLCO1B3 induction in cancerous versus healthy tissue $\mathrm{e}^{1,2}$. Our laboratory and others have investigated the role of steroid hormone transporters in modulating intratumoral androgen concentrations that promote CRPC progression ${ }^{1-5}$. We showed that the organic anion polypeptide 1B3 (OATP1B3) transporter facilitates the diffusion of unconjugated testosterone into cells, and polymorphic variations in the SLCO1B3 gene (encoding OATP1B3) are related to both the progression-free survival of men receiving ADT for hormone-responsive prostate cancer and the overall survival of men with CRPC $^{3,4}$. We subsequently showed de novo OATP1B3 expression in prostate tumor cells contributes to greater androgen uptake, which is consistent with its role in disease progression ${ }^{1}$.

Because OATP1B3 is an important predictive marker in men with prostate cancer, it is essential to understand mechanisms governing its de novo expression. Transcriptional regulatory mechanisms for SLCO1B3 expression include the involvement of different transcription factors and is highly dependent on tissue types. The SLCO1B3 promoter can be transactivated by the farneosid X receptor (FXR) ${ }^{6}$, hepatic nuclear factor- $1 \alpha^{7}$, and STAT5 ${ }^{6}$, while the hepatocyte nuclear factor $3 \beta^{8}$, and constitutive androstane receptor ${ }^{9}$ have been reported to repress

\footnotetext{
${ }^{1}$ Molecular Pharmacology Section, Genitourinary Malignancies Branch, Center for Cancer Research, National Cancer Institute, National Institutes of Health, 9000 Rockville Pike, Building 10, Room 5A03, Bethesda, MD 20892, USA. ${ }^{2}$ Clinical Pharmacology Program, Center for Cancer Research, National Cancer Institute, National Institutes of Health, Bethesda, MD 20892, USA. ${ }^{\circledR}$ email: figgw@mail.nih.gov
} 
transcription. Hypoxia-mediated ${ }^{10,11}$ and epigenetic mechanisms such as DNA methylation ${ }^{10,12-14}$ can also play a significant role in $S L C O 1 B 3 \mathrm{mRNA}$ expression.

The transcriptional regulation of SLCO1B3 in prostatic tissue has not been fully elucidated. We have recently discovered that chetomin is a potent inducer of SLCO1B3 transcription in prostate cancer cells ${ }^{1}$. Chetomin targets p300 (E1A binding protein), a global transcriptional coactivator, by disrupting the structure of its CH1 domain and precludes its interaction with the hypoxia-inducible factor- $1 \alpha$ (HIF- $1 \alpha$ ) transcription factor. We found that modulation of HIF-1 1 only modestly increased SLCO1B3 expression, suggesting that expression of SLCO1B3 may occur via a p300-mediated regulatory mechanism ${ }^{1}$. Previous studies have shown that $\mathrm{p} 300$ increases during $\mathrm{ADT}$ and that this upregulation is associated with increased tumor growth and progression; conversely, increasing androgens produced a dose-dependent decrease in p300 expression ${ }^{15}$.

MicroRNAs (miRNAs) are small noncoding RNA molecules that function as key post-transcriptional regulators of gene expression by promoting mRNA degradation or translational repression ${ }^{16}$. The regulation of drug transporters by miRNAs has recently been investigated for the human intestine ${ }^{17}$. Though little research has involved miRNA in the direct regulation of SLCO1B3 in prostate cancer, SLCO1B3 was found to be negatively correlated with rifampin-induced miRNA expression in hepatocytes ${ }^{18}$. In addition, FXR and its target genes including SLCO1B3 are regulated by hsa-miR-192, also in hepatocytes ${ }^{19}$. More broadly, miRNA involvement in prostate cancer initiation, proliferation, progression, and therapeutic resistance has been relatively well described, with thousands of relevant miRNA species characterized. miRNAs have been found to regulate key driving pathways in prostate cancer, such as the AR signaling axis, TMPRSS2-ERG, and PTEN ${ }^{20-23}$.

In this study, we investigated the effect of the second-generation anti-androgen abiraterone on SLCO1B3 expression. We found that abiraterone stimulated the expression of SLCO1B3 and the mechanism of this effect involved regulation by miRNAs.

\section{Results}

Abiraterone treatment and increased SLCO1B3 expression in prostate cancer cells. The relationship between p300/CBP and ADT has been characterized in advanced prostate cancer ${ }^{15,24}$. Since p300-mediates expression of $S L C O 1 B 3$, we first examined the effect of antiandrogens on SLCO1B3 expression in two well characterized AR-positive prostate cancer cell lines (22Rv1 \& LNCaP). Cells were treated with the second-generation antiandrogens abiraterone and enzalutamide, as well as finasteride, for $24 \mathrm{~h}$. In LNCaP cells, abiraterone, enzalutamide and finasteride modestly decreased SLCO1B3 mRNA levels (Fig. 1a). Enzalutamide had a modest effect on transcript levels in 22Rv1 cells (Fig. 1b). Surprisingly, treatment with $20 \mu \mathrm{M}$ abiraterone produced a significant increase in SLCO1B3 expression in 22Rv1 cells at $24 \mathrm{~h}$ (Fig. 1b), and this inhibition was sustained up to $72 \mathrm{~h}$ of treatment (Supplementary Fig. S1).

Since a subset of patients is inherently resistant to both abiraterone and enzalutamide due to expression of $\mathrm{AR}-\mathrm{V} 7^{25}$, we next examined the mechanism of abiraterone-induced upregulation of SLCO1B3 expression in $22 \mathrm{Rv} 1$ cells as a possible mechanism of resistance since this cell line expresses high levels of endogenous AR-variants (e.g., AR-V7) and exhibits intrinsic resistance to both enzalutamide and abiraterone ${ }^{26,27}$. When cells were treated with varying concentrations of abiraterone, expression of SLCO1B3 transcript was significantly upregulated in a dose-dependent manner (Fig. 1c). To investigate whether androgen mediated SLCO1B3 upregulation, we treated $22 \mathrm{Rv} 1$ cells in phenol red-free, charcoal-stripped media containing abiraterone in the presence and absence of two androgens, testosterone $(\mathrm{T})$ and dihydrotestosterone (DHT). We found that SLCO1B3 was significantly upregulated by abiraterone regardless of androgen stimulation (Fig. 1d,e). While the abiraterone + DHT treatment appears to have induced SLCO1B3 to a lesser extent than ABI treatment alone, there is not a statistically significant difference in the SLCO1B3 expression levels between two treatment groups. Treatment with T or DHT increased KLK3 mRNA expression (data not shown), indicating androgen stimulation was potent enough to induce AR-dependent gene expression, but neither increased SLCO1B3 expression. We also evaluated whether there may be a dose-dependent effect with enzalutamide on SLCO1B3 transcripts and found that treatment with $10 \mu \mathrm{M}$ and $20 \mu \mathrm{M}$ doses of enzalutamide in the presence and absence of DHT did not affect the expression of SLCO1B3 (Fig. 1e).

Since LNCaP cells express the AR-T878A point mutation and 22Rv1 cells express both full-length and a constitutive active AR splice variant (AR-V7), we next determined the effect of abiraterone on the androgenresponsive $\mathrm{VCaP}$ cell line, which harbors the wild-type $\mathrm{AR}$ and $\mathrm{AR}-\mathrm{V}^{28}$. We showed that abiraterone also increased the expression of SLCO1B3 mRNA in VCaP cells in a dose-dependent manner similar to that observed in $22 \mathrm{Rv} 1$ cells (Fig. 1f).

Other CYP17A1 inhibitors upregulate SLCO1B3. Since abiraterone is a potent inhibitor of the cytochrome P450 17A1 enzyme (CYP17), we next determined whether other CYP17A1 inhibitors may also be involved in regulating SLCO1B3 expression. $22 \mathrm{Rv} 1$ cells were treated with $20 \mu \mathrm{M}$ ketoconazole, $10 \mu \mathrm{M}$ seviteronel, and $10 \mu \mathrm{M}$ galeterone alongside $20 \mu \mathrm{M}$ abiraterone to assess whether upregulation of SLCO1B3 is abiraterone-specific, or whether this effect is common to the CYP17A1 inhibitor drug class. All drugs tested (except seviteronel) induced a statistically significant increase in SLCO1B3 transcripts, with abiraterone producing the largest increase of SLCO1B3 expression (Fig. 2).

Abiraterone upregulates expression of tumor SLCO1B3 in a 22Rv1 mouse xenograft model. To assess whether abiraterone could upregulate SLCO1B3 in vivo, we treated mice bearing 22Rv1 xenografts with abiraterone acetate or vehicle control for either 5 or 15 days. As anticipated abiraterone treatment did not reduce the growth of $22 \mathrm{Rv} 1$ xenografts (data not shown), consistent with previous studies ${ }^{26,29}$. After 5 days of abiraterone acetate treatment, tumors were excised and digested to measure mRNA transcripts. Abira- 
(a)

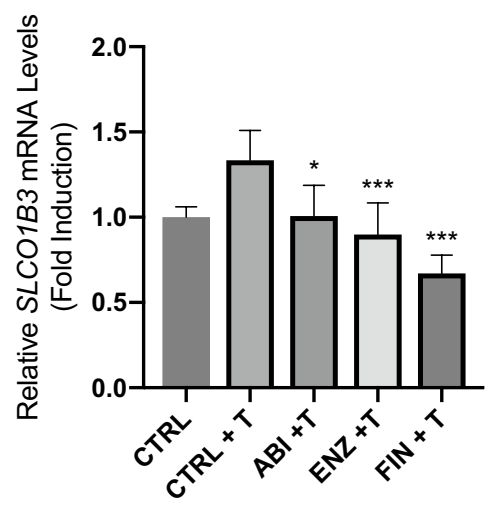

(d)

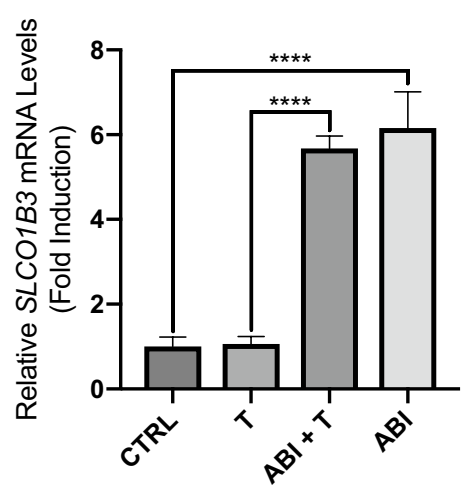

(b)

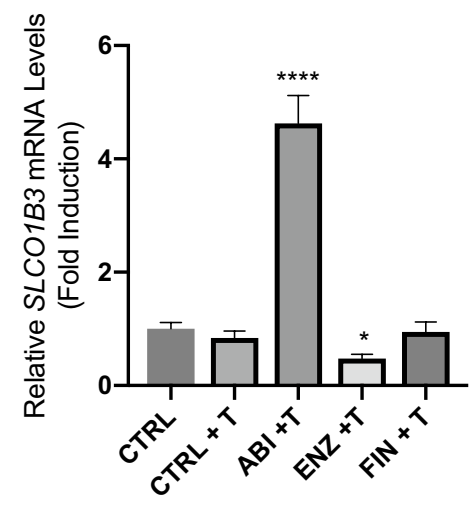

(e)

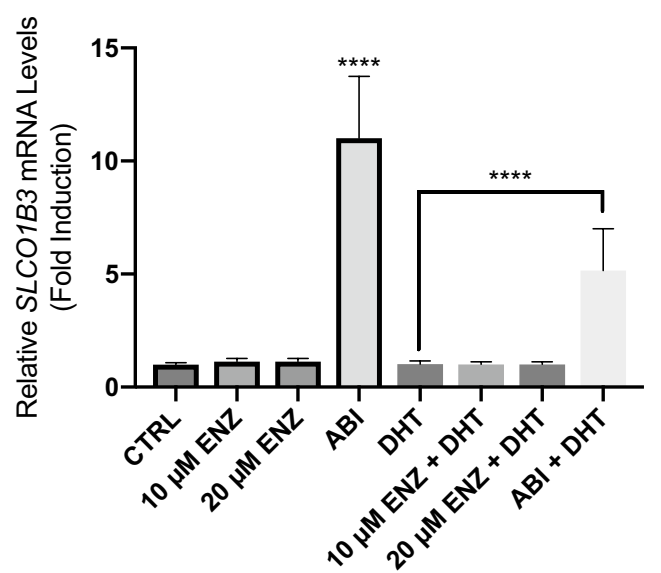

(c)

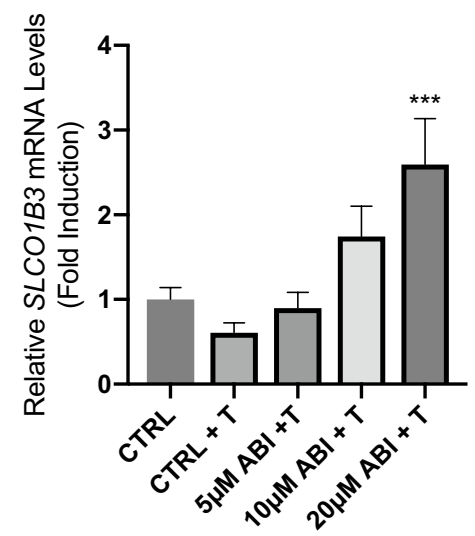

(f)

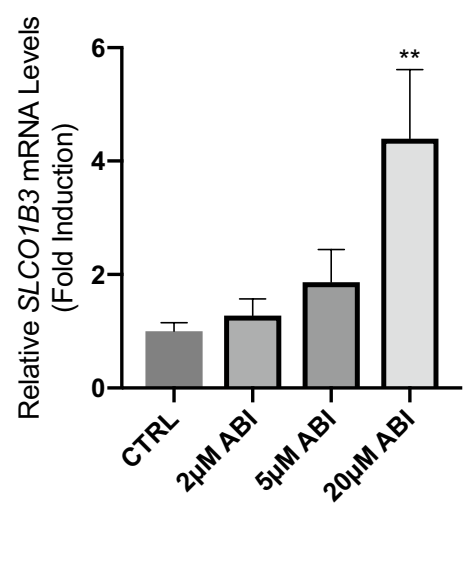

Figure 1. AR-positive prostate cancer cell lines ( $\mathrm{LNCaP}, 22 \mathrm{Rv} 1$, and $\mathrm{VCaP}$ ) were treated with $1 \mathrm{nM}$ testosterone and three prostate cancer drugs abiraterone (ABI), enzalutamide (ENZ), or finasteride (FIN) for $24 \mathrm{~h}$. RNA was extracted and assessed for changes in SLCO1B3 expression using qPCR. Gene expression was normalized to expression of $\beta$-actin. (a) All treatment groups produced a significant reduction in its expression at $72 \mathrm{~h}$ in LNCaP Cells. (b) Abiraterone produced a highly significant upregulation of SLCO1B3 in $22 \mathrm{Rv} 1$ cells. (c) Concentration-dependent effect of abiraterone (ABI) significantly increased the expression of SLCO1B3. 22Rv1 cells were treated in phenol red-free, charcoal-stripped media with abiraterone (ABI), enzalutamide (ENZ) in the presence or absence of (d) $1 \mathrm{nM}$ testosterone (T) or (e) $1 \mathrm{nM}$ dihydrotestosterone (DHT) for $24 \mathrm{~h}$. 22Rv1 cells were also treated with a higher dose $(20 \mu \mathrm{M})$ of ENZ to confirm that ENZ treatment has no effect on SLCO1B3 expression. (f) The AR-positive prostate cancer cell line, VCaP, was treated with abiraterone at increasing concentrations for $24 \mathrm{~h}$. ABI increased SLCO1B3 in a dose-dependent manner. RNA was harvested from cells after $24 \mathrm{~h}$ of treatment and assessed for changes in SLCO1B3 expression using qPCR analysis. Gene expression was normalized to expression of $\beta$-actin. Treatment in AR-negative PC3 cells did not affect SLCO1B3 expression (data not shown). Statistical tests were performed against CRTL + T for A-C. These data are the result of three independent experiments. ${ }^{\star} \mathrm{P}<0.05,{ }^{\star *} \mathrm{P}<0.001,{ }^{* *} \mathrm{P}<0.0001,{ }^{\star * *} \mathrm{P}<0.00001$.

terone-treated tumors exhibited a 2.5-fold increase in SLCO1B3 mRNA expression when compared with vehicle control $(\mathrm{p}<0.05)$ (Fig. 3a). After 15 days of treatment, abiraterone-treated mice had a similar upregulation of SLCO1B3 ( $\mathrm{p}<0.05)$ when compared with control mice treated with vehicle control. Levels of slco1b2 (the mouse analogue of human SLCO1B3) in the livers of the mice were modestly decreased at day 15 of treatment (Fig. 3b).

Abiraterone-induced overexpression of SLCO1B3 is mediated by miRNA expression. We have previously demonstrated that the liver-type SLCO1B3 (lt-SLCO1B3) was predominantly overexpressed in prostate tumor tissues ${ }^{1}$. We next determined whether abiraterone-induced SLCO1B3 mRNA expression occurred at the promoter level. Analysis of the SLCO1B3 promoter (containing the full-length 1492 bp upstream promoter from the transcription start site) characterized by luciferase reporter assays showed no cis-regulatory response to abiraterone treatment (Supplementary Fig. S2). We therefore hypothesized that the mechanism driving abiraterone-induced upregulation of SLCO1B3 was post-transcriptional, likely mediated by miRNA. To investigate the relationship between miRNA and $l t-S L C O 1 B 3$ expression, we profiled the miRNA transcriptome of $22 \mathrm{Rv} 1$ cells 


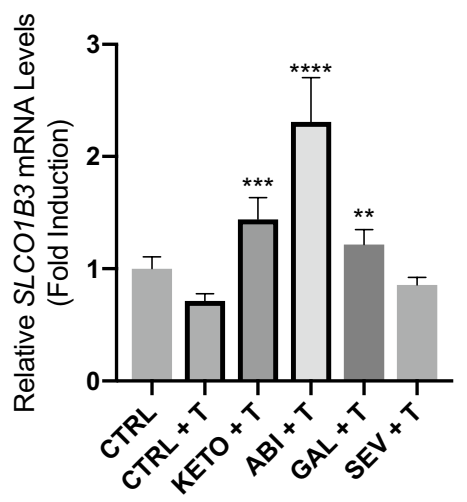

Figure 2. The effect of CYP inhibitors on SLCO1B3 expression. 22Rv1 cells were treated with four CYP17 inhibitors, ketoconazole (Keto), abiraterone (ABI), galeterone (GAL), or seviteronel (SEV), in media supplemented with $1 \mathrm{nM}$ testosterone for $24 \mathrm{~h}$. Cells were treated with ketoconazole and abiraterone at a concentration of $20 \mu \mathrm{M}$ and galeterone and seviteronel at a dose of $10 \mu \mathrm{M}$ based on precedents set for in vitro experiments in previous publications. RNA was extracted and assessed for changes in SLCO1B3 expression using qPCR. Gene expression was normalized to expression of $\beta$-actin. All CYP17 inhibitors but seviteronel produced statistically significant induction of SLCO1B3 in 22Rv1 cells. Statistical tests were performed against CRTL + T. Data represent three independent experiments with triplicate measurements. ${ }^{*} \mathrm{P}<0.001$,

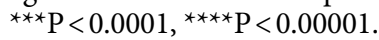

(a)

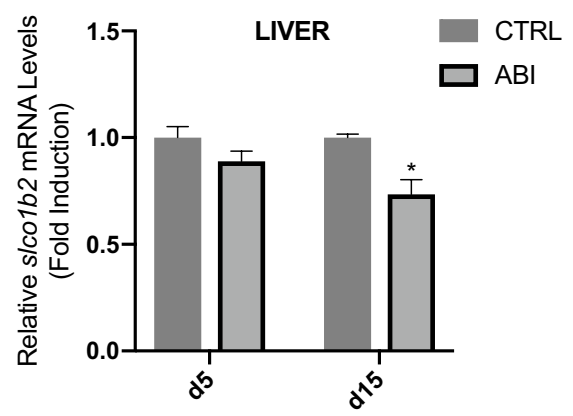

(b)

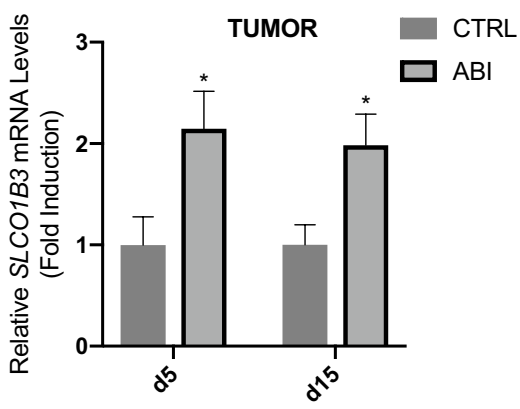

Figure 3. Mice bearing $22 \mathrm{Rv} 1$ prostate cancer xenografts were treated with abiraterone acetate (AA) at $5 \mathrm{mmol} /$ $\mathrm{kg} /$ day or vehicle control (95\% safflower oil, $5 \%$ benzyl alcohol) via intraperitoneal injection every day for either (a) 5 or (b) 15 days. RNA was extracted from harvested tumor or liver tissue and reverse transcribed for analysis by qPCR. Gene expression was normalized to expression of $\beta$-actin. Significant upregulation of SLCO1B3 was observed at both time points. Mouse livers were assessed for changes in slcolb2 (the mouse analogue of human SLCO1B3) expression. While AA treatment induced a statistically significant decrease in mouse liver slco1b2 expression, the magnitude of the change was small and may not reflect how SLCO1B3 expression in a human liver would be affected by AA treatment. ${ }^{\star} \mathrm{P}<0.05$.

treated with $20 \mu \mathrm{M}$ abiraterone for $24 \mathrm{~h}$, using the NanoString nCounter Human v3 miRNA panel. This assay rapidly and efficiently profiles 800 highly curated human miRNAs. We chose this hybridization-based nucleic acid counting method to avoid amplification biases, at the expense of resolving low-abundance miRNA species. Three miRNA species hsa-miR-579-3p, hsa-miR-16-5p, and hsa-mir-181a-5p were identified as significantly differentially expressed, demonstrating greater than 1.5 -fold change $(\mathrm{P}<0.05)$ (Fig. $4 \mathrm{a}$ and Supplementary Fig. S3).

To predict miRNA species with stable expression under abiraterone treatment, globally normalized counts from the NanoString panel were input in RefFinder, a program which aggregates stability-ranking programs BestKeeper, NormFinder, Genorm, and the comparative $\Delta$-Ct method by taking the geometric mean of each program's generated rankings. Hsa-let-7e-5p, hsa-miR-32-5p, and hsa-miR-148b-3p were predicted to be the most stable potential reference genes (Table 1). Stability of these reference genes was validated by qPCR analysis. As it was ranked as most stable by RefFinder, hsa-let-7e-5p was selected as the reference species against which the selected miRNA species would be measured, although use of hsa-miR-32-5p and hsa-miR-148b-3p provided similar results.

MiRNA species identified as significantly differentially expressed by the NanoString panel were then screened by TargetScan $3.0^{30}$ and miRanda software ${ }^{31}$, miRNA-mRNA binding domain alignment programs. 
(a)

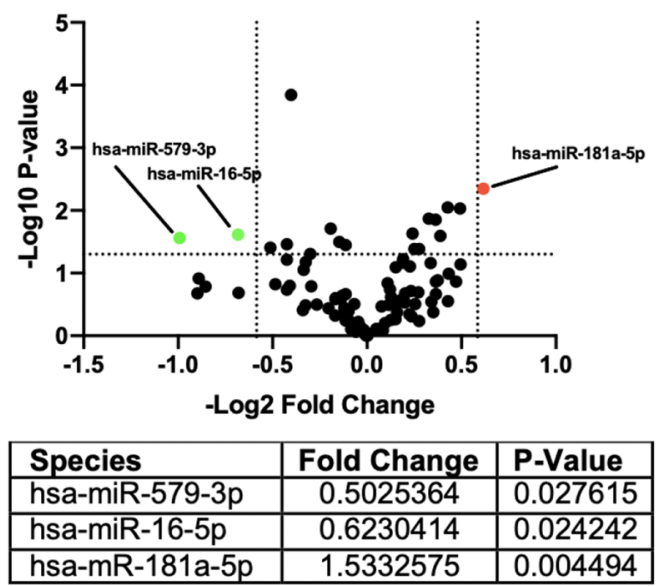

(b)

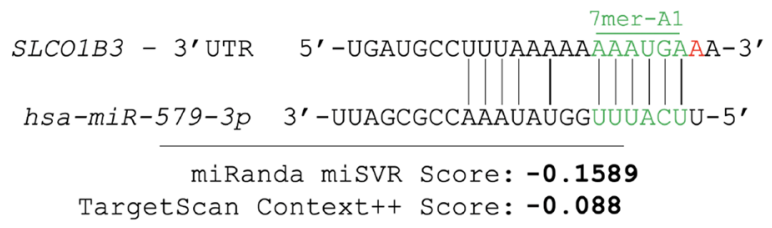

Figure 4. To investigate the relationship between miRNA and $l t-S L C O 1 B 3$ expression, we profiled the miRNA transcriptome of cells treated with abiraterone using NanoString. (a) Differentially expressed miRNA species above 1.5-fold change and $\mathrm{P}<0.05$ in an unpaired Student's T-test were selected for further analysis. (b) miRNA-mRNA binding prediction programs miRanda and TargetScan identified binding between hsa-miR579-3p and SLCO1B3. Images show binding of hsa-miR-579-3p to the promoter of SLCO1B3 using TargetScan and miRanda.

\begin{tabular}{|l|l|l|l|l|l|l|}
\hline Rank & miRNA species & Delta CT & BestKeeper & NormFinder & Genorm & Geomean of ranking \\
\hline 1 & hsa-let-7e-5p & 1 & 2 & 2 & 1 & 1.41 \\
\hline 2 & hsa-miR-32-5p & 2 & 1 & 1 & 21 & 2.55 \\
\hline 3 & hsa-miR-148b-3p & 3 & 3 & 3 & 22 & 4.94 \\
\hline 4 & hsa-miR-592 & 5 & 5 & 4 & 23 & 7 \\
\hline 5 & hsa-miR-361-5p & 4 & 4 & 7 & 23 & 7.12 \\
\hline 6 & hsa-miR-582-5p & 6 & 7 & 10 & 25 & 10.12 \\
\hline 7 & hsa-miR-9-5p & 8 & 11 & 6 & 26 & 10.82 \\
\hline 8 & hsa-miR-99a-5p & 11 & 10 & 9 & 27 & 12.79 \\
\hline 9 & hsa-miR-98-5p & 7 & 9 & 14 & 36 & 13.35 \\
\hline 10 & hsa-miR-22-3p & 9 & 6 & 16 & 36 & 13.37 \\
\hline
\end{tabular}

Table 1. RefFinder ranking of miRNA Species.

Only hsa-miR-579-3p was predicted by TargetScan and miRanda to bind to the 3 '-UTR of the lt-SLCO1B3 transcript (Fig. 4b). The Context++ score generated by TargetScan7.2 is output from a model incorporating 14 miRNA-binding parameters and is reflective of the probability and extent to which an miRNA species will repress expression of a given mRNA target ${ }^{30}$. The Context++ score of -0.088 given to the hsa-miR-579-3p/ SLCO1B3-3'UTR is in the 92nd percentile for predicted SLCO1B3 3'UTR-binding miRNA species. The miRanda program is a machine learning-based predictive binding algorithm, and the calculated score of -0.1589 reflects an approximate $40-50 \%$ probability that hsa-miR-579-3p represses transcript level of SLCO1B3 to the extent which we have observed, which is approximately $30-50 \%{ }^{32}$.

The effects of abiraterone treatment on the expression of $l t-S L C O 1 B 3$ and hsa-miR-579-3p in the samples processed for NanoString analysis were further validated by qPCR. Recapitulating the NanoString results, qPCR 
(a)

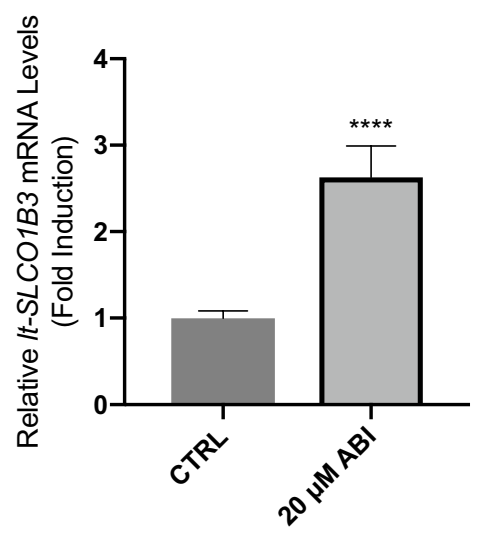

(b)

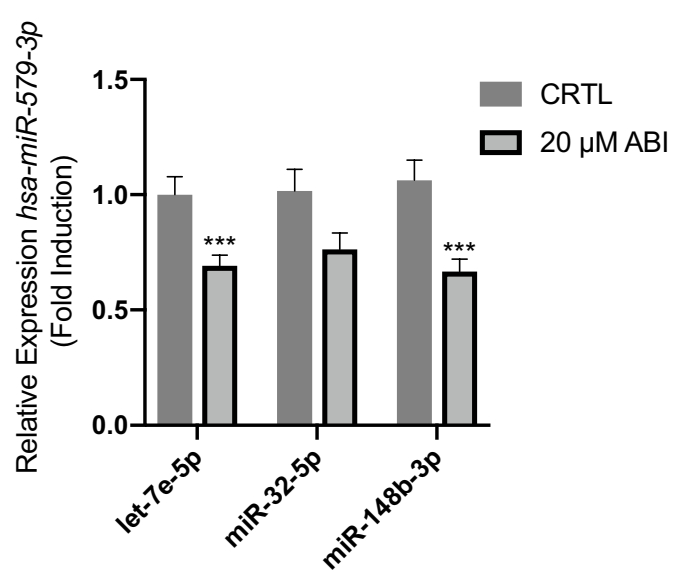

(c)

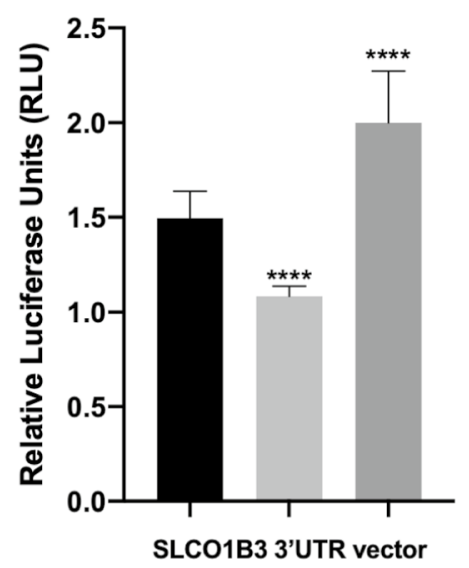

- Control

hsa-miR-579-3p mimic

Abiraterone

Figure 5. Abiraterone induces overexpression of $l t$-SLCO1B3 while suppressing hsa-miR-579-5p expression. Effects of abiraterone treatment on the expression of (a) $l t-S L C O 1 B 3$ and (b) hsa-miR-579-3p in the samples processed for NanoString analysis were validated by qPCR analysis. As expected hsa-miR-579-3p expression was reduced and $l t-S L C O 1 B 3$ expression was increased following abiraterone treatment, consistent with miRNAtranscript repression. (c) hsa-miR-579-3p directly binds to SLCO1B3 3'UTR. Co-transfection of 22Rv1 cells was performed with the 3'UTR reporter plasmid (or vector control plasmid) and hsa-miR-579-3p mimic (or negative control mimic) for $24 \mathrm{~h}$ followed by luciferase reporter assays. The assay revealed a significant decrease in SLCO1B3 reporter activity in the presence of the mimic. 22Rv1 cells were also transfected with the 3'UTR plasmid (or vector control plasmid) followed by treatment with $20 \mu \mathrm{M}$ Abiraterone (or vehicle DMSO control) for $24 \mathrm{~h}$. Abiraterone treatment resulted in an increase in SLCO1B3 luciferase reporter activity. No significant change was observed in the vector control plasmid lacking the $3^{\prime} U T R$ (see Supplementary Fig. S4). These data are the results of three independent experiments performed in triplicates. ${ }^{\star *}{ }^{*} \mathrm{P}<0.005,{ }^{\star * * *} \mathrm{P}<0.0001$.

analysis found hsa-miR-579-3p miRNA expression to be reduced and $l t$-SLCO1B3 mRNA expression increased following abiraterone treatment (Fig. 5a,b). This is consistent with miRNA-mediated repression of $l t-S L C O 1 B 3$. Using luciferase reporter system, we further demonstrated that hsa-miR-579-3p indeed binds to the SLCO1B3 3'UTR by cloning this sequence into a psiCHECK-2 vector, containing the renilla and continuously expressed firefly luciferase reporter gene. Next, we performed co-transfections of the psiCHECK-2-SLCO1B3 3'UTR plasmid (or empty vector plasmid) together with the hsa-miR-579-3p mimic or nonspecific mimic (negative control) in $22 \mathrm{Rv} 1$ cells followed by assaying for luciferase activity. Dual-luciferase reporter assay verified that SLCO1B3 is a target gene of hsa-miR-579-3p as our data demonstrated a significant decrease in luciferase reporter activity when cells were treated with the hsa-miR-579-3p mimic in comparison to the negative control (Fig. 5c), indicating that hsa-miR-579-3p directly targets $S L C O 1 B 3$ by binding to its $3^{\prime} \mathrm{UTR}$. We also showed that no significant difference was observed in cells transfected with a psiCHECK-2 vector lacking the $3^{\prime}$-UTR, further confirming the specific hsa-miR-579-3p binding interaction with the 3'UTR of SLCO1B3 (Supplementary Fig. S4). Luciferase activity was recovered and further increased in cells transfected with the psiCHECK-2-SLCO1B3 3' UTR plasmid and treated with abiraterone as compared to the vehicle DMSO treated control, demonstrating that abiraterone treatment decreased hsa-miR-579-3p miRNA expression, resulting in increased SLCO1B3 reporter activity (Fig. $5 \mathrm{c}$ and Supplementary Fig. S4). These results directly support our findings from both NanoString 
and qPCR analyses that hsa-miR-579-3p binds to the SLCO1B3 3'UTR and provide additional evidence for a mechanism of abiraterone-induced SLCO1B3 expression mediated by hsa-miR-579-3p.

\section{Discussion}

We have shown that abiraterone, one of the most commonly prescribed drugs for CRPC, induces a significant upregulation of SLCO1B3 transcripts in 22Rv1 and VCaP cells and in mice bearing 22Rv1 tumor xenografts. Expression levels of total-SLCO1B3 expression increase with abiraterone treatment in a dose-dependent manner in both $22 \mathrm{Rv} 1$ and $\mathrm{VCaP}$ cells. We demonstrated that abiraterone-mediated increase in SLCO1B3 transcripts occurred independently of androgen stimulation, consistent with our finding that abiraterone treatment in the androgen-independent PC3 cell line had no effect on SLCO1B3 expression (data not shown). We have previously shown that PC 3 cells have higher endogenous expression of SLCO1B3 than $22 \mathrm{Rv} 1$ cells $^{1}$ and have low level expressions of the CYP17A1 enzyme; therefore, these cells may not be as responsive to the effect of abiraterone treatment ${ }^{33}$.

We further demonstrated that other CYP17A1 inhibitors (abiraterone, ketoconazole, and galeterone) can upregulate SLCO1B3 transcripts. Abiraterone, ketoconazole, and galeterone inhibit both 17a-hydroxylase and 17,20-lyase of CYP17A1 whereas seviteronel has selective inhibition of 17,20-lyase over 17a-hydroxylase ${ }^{34,35}$. Whether 17,20-lyase selectivity or potency can explain why seviteronel does not affect SLCO1B3 remains to be determined ${ }^{36}$.

We showed that the mechanism of abiraterone-induced SLCO1B3 expression is regulated by miRNAs (hsamiR-579-3p, hsa-miR-16-5p, and hsa-mir-181a-5p). We used two different miRNA target prediction tools to determine which of the three miRNAs interact with SLCO1B3. Using this computational approach, we predicted binding of hsa-miR-579-3p to the 3'-UTR of the SLCO1B3. Validation experiments were subsequently performed to confirm the NanoString data and revealed that the increase in abiraterone-mediated SLCO1B3 transcripts correlated with a decrease in expression of hsa-miR-579-3p, as verified with luciferase reporter assays demonstrating the binding of hsa-miR-579-3p to the SLCO1B3 3'UTR. Hsa-miR-579-3p has previously been shown to be downregulated in exosomes secreted by prostate cancer cells under hypoxic conditions, and is otherwise implicated in tumorigenesis in multiple other cell types via modulation of oncogenic kinase signaling pathways, such as PI3K, BRAF, RAS, and AKT ${ }^{37-40}$.

Previous studies have reported that $S L C O 1 B 3$ can be regulated by miRNAs. In a study evaluating membrane drug transporters and miRNA gene expression changes mediated by rifampin treatment in hepatocytes, SLCO1B3 expression was reduced after rifampin treatment and negatively correlated with rifampin-induced hsa-miR-92a expression $^{18}$. In addition, hsa-miR-192 was found to inhibit FXR, a known regulator of SLCO1B3, which in turn, suppressed SLCO1B3 expression ${ }^{19,41}$.

A recent study by Zedan et al. found hsa-miR-141-3p and hsa-miR-375-3p to be of clinical significance in predicting the outcome of patients treated with abiraterone. The study findings were based on analysis of a preselected group of five miRNAs that came from a literature review rather than using a global miRNA profiling approach like NanoString; thus, this may explain the discrepancy in their results, in addition to patient heterogeneity and exposure to prior treatment regimens ${ }^{42}$. Our inability to detect significant differential expression of these species is likely due to the difference in model systems, where we used an in vitro cell line model (a limitation of our study) rather than patient samples. Future studies using clinical samples would be needed to confirm whether hsa-miR-579-3p can be identified in patients treated with abiraterone and to correlate this observation with SLCO1B3 expression. It also remains to be determined the effect of abiraterone-induced SLCO1B3 expression on tissue abiraterone levels since the drug has been hypothesized to be an SLCO substrate ${ }^{43}$. Our study is further limited by the use of $22 \mathrm{Rv} 1$ cells with intrinsic resistance to abiraterone; future studies should examine the effect of miRNA-mediated SLCO1B3 expression in cell lines with acquired resistance to the drug as well as subsequent transport of substrates.

Our study identified two miRNAs, hsa-miR-16-5p and hsa-mir-181a-5p, that were differentially regulated by abiraterone treatment. Both miRNAs have previously been identified as potential diagnostic and therapeutic biomarkers for prostate cancer ${ }^{44-49}$. Interestingly, hsa-miR-181a-5p is known to suppress GRP78 in $22 \mathrm{Rv1}$ cells $^{50}$. GRP78, coordinates with SIAH2 to degrade AR-V7 ${ }^{51}$. Induction of hsa-miR-181a-5p could therefore be another miRNA-mediated mechanism of resistance against abiraterone. Therefore, while these two miRNAs may not be involved in regulating $S L C O 1 B 3$ as a potential mechanism for abiraterone resistance, they may contribute to the overall abiraterone resistance mechanism and future studies are warranted to investigate their relevance.

In summary, we have shown that CYP17 inhibitors can stimulate the expression of SLCO1B3, the known testosterone uptake transporter. We demonstrated that the mechanism of abiraterone-induced SLCO1B3 expression is mediated by the miRNA, hsa-miR-579-3p. To our knowledge, this is the first report of a mechanism of resistance of abiraterone treatment attributed to miRNA regulation in controlling the expression of OATP1B3, an androgen transporter that has been implicated in driving the resistance to ADT through the mechanism of increasing uptake of residual androgens into prostate tumors. In addition, hsa-miR-579-3p may serve as a potential therapeutic biomarker for abiraterone resistance.

\section{Materials and methods}

Cell culture. All prostate cancer cell lines (CWR22Rv1, LNCaP, PC3, and VCaP) were purchased from ATCC (Manassas, VA). Cell culture reagents were obtained from Gibco/Thermo Fisher Scientific (Gaithersburgh, MD), unless otherwise specified. The 22Rv1 and LNCaP cell lines were maintained in phenol red-free RPMI 1640 Medium supplemented with 10\% fetal bovine serum (FBS, Atlanta Biologicals, Flowery Branch, GA), 100 units $/ \mathrm{mL}$ penicillin, 100 units/mL streptomycin, and $0.25 \mu \mathrm{g} / \mathrm{mL}$ amphotericin $\mathrm{B}$. For all experiments involving addition of steroid hormone, cells were plated in maintenance media, then medium was replaced with 
RPMI Medium 1640 supplemented with 10\% charcoal stripped FBS (all other media components remained unchanged) for approximately $7 \mathrm{~h}$ before treatment. PC3 cells were cultured in F-12K Nutrient Mixture medium (Gibco), and VCaP cells were maintained and treated in DMEM Medium (Gibco) supplemented with 10\% FBS, 100 units $/ \mathrm{mL}$ penicillin, 100 units $/ \mathrm{mL}$ streptomycin, and $0.25 \mu \mathrm{g} / \mathrm{mL}$ amphotericin $\mathrm{B}$.

Reagents. Abiraterone and enzalutamide were purchased from Selleckchem (Houston, TX). Galeterone, finasteride, and ketoconazole were purchased from Sigma-Aldrich (St. Louis, MO). VT-646 (seviteronel) was ordered from Chemscene (Monmouth Junction, NJ). Abiraterone acetate for xenograft studies was purchased from Medkoo Biosciences (Morrisville, NC). All drugs were dissolved in DMSO, aliquoted and stored at $-80^{\circ} \mathrm{C}$. Testosterone (Sigma) was dissolved in ethanol and DHT (Steraloids Inc., Newport, RI) was dissolved in DMSO.

Semiquantitative real-time polymerase chain reaction. $22 \mathrm{RV} 1$ and LNCaP cells were plated in 6 -well dishes in maintenance media the day before treatment. Cells were serum-starved approximately $7 \mathrm{~h}$ prior to treatment and then incubated with the treatment medium for $24 \mathrm{~h}$ or $72 \mathrm{~h}$. Total RNA was extracted from cells using the QIAshredder and RNeasy mini kits (Qiagen) as per the manufacturer's protocol. Extracted RNA was then assessed for purity using a NanoDrop spectrophotometer (Molecular Devices) and all samples were diluted to a concentration of $80 \mathrm{ng} / \mu \mathrm{L}$ in RNase-free water. Using $12 \mu \mathrm{L}$ of each of these stocks, cDNA was synthesized using the SuperScript III First-Strand Synthesis System for RT PCR (Thermofisher) as per the manufacturer's protocol.

The RT PCR reaction yields $30 \mu \mathrm{L}$ of cDNA reaction product. Two microliters of this cDNA was amplified by qPCR using the following TaqMan probes: SLCO1B3 (Hs00251986_m1, Applied Biosystems), LT-SLCO1B3 (Hs01127179_mH, Applied Biosystems). For each qPCR reaction, $2 \mu \mathrm{L}$ of cDNA was mixed with $10 \mu \mathrm{L}$ of Taqman Gene Expression Master Mix (Applied Biosystems), $7 \mu \mathrm{L}$ of RNAse-free water, and $1 \mu \mathrm{L}$ of the respective Taqman qPCR primer. Semiquantitative Real-Time Polymerase Chain Reaction (qPCR) was performed using an Applied Biosystems StepOnePlus Real-Time PCR system with StepOne Software. Each qPCR reaction was run in triplicate and gene expression was normalized to expression of $\beta$-actin. Fold-change in gene expression was calculated using the $\Delta \Delta \mathrm{C}_{\mathrm{t}}$ method as described in the SABiosciences 2009 RT2 Profiler PCR Array System User Manual (SABiosciences, Frederick, MD).

Prostate cancer xenograft study. Six-week old, male, severe combined immunodeficiency (SCID) mice were obtained from the NCI-Frederick Animal Production Area. 22Rv1 cells were cultured in maintenance media and harvested when they reached $80 \%$ confluency. Cells were washed with sterile phosphate buffered saline (Gibco) and approximately 6 million cells were injected subcutaneously into the rear flank of each SCID mouse. Mice were monitored and weighed daily. When tumor volumes reached $\sim 200-250 \mathrm{~mm}^{3}$, animals were randomized into two groups of control vs treatment $(n=5)$. Each group was treated daily with intraperitoneal (i.p.) bolus injections of either the drug vehicle (95\% safflower oil, $5 \%$ benzyl alcohol) or abiraterone acetate $(0.5 \mathrm{mmol} / \mathrm{kg})$ for either 5 or 15 consecutive days $\mathrm{s}^{52}$. Tumor measurements used to calculate tumor volume (tumor volume $=$ tumor length ${ }^{\star}$ tumor width ${ }^{\star}$ tumor height ${ }^{\star} \pi / 6$ ) were taken three days per week and mice were weighed daily. Mouse livers and tumors were excised on either day 5 or day 15 of treatment, and harvested tissue samples were snap frozen in liquid nitrogen for subsequent gene expression analysis.

To analyze gene expression in the liver and tumor tissues, tissue was thawed on ice and an approximately $30 \mathrm{mg}$ section was shaved off using a scalpel. This section was placed in a bead homogenizer tube with $600 \mu \mathrm{L}$ of buffer RLT (Qiagen, Hilden, Germany) and homogenized for roughly $30 \mathrm{~s}$ (BeadBug Benchtop Homogenizer, Benchmark Scientific). After the tissue was homogenized in lysis buffer, an equal $(600 \mu \mathrm{L})$ volume of $70 \%$ alcohol was added to the tube and mixed gently to precipitate RNA. Then, $700 \mu \mathrm{L}$ of the precipitated RNA mixture was transferred to an RNeasy Mini spin column (Qiagen) and the RNA extraction proceeded according to the RNeasy Mini Kit protocol (Qiagen). RNA was reverse-transcribed to cDNA and used for qPCR analysis using Mouse ACTB (4352663, Applied Biosciences) and slco1b2 (Mm00451510_m1, Applied Biosystems) probes. qPCR and data analysis were performed as previously described.

The National Cancer Institute (NCI) is accredited by the Association for Assessment and Accreditation of Laboratory Animal Care (AAALAC) International and follows the Public Health Service (PHS) Policy for the Care and Use of Laboratory Animals. Animal care was provided in accordance with the Guide for the Care and Use of Laboratory Animals. The study protocol was approved by the NCI Animal Care and Use Committee. This study was carried out in compliance with the ARRIVE guidelines.

miRNA analysis. miRNA extraction. 22Rv1 cells were homogenized using a QIAshredder kit (Qiagen Cat\#: 79654) and total RNA was extracted from the homogenate with a miRNeasy Mini Kit (Qiagen Cat\#: 217004) following manufacturer protocols. RNA was quantified for normalization using a NanoDrop ND-1000 spectrophotometer (Thermo Scientific).

miRNA profiling. Total RNA was prepared for and profiled using the nCounter Human v3 miRNA Panel (NanoString Technologies) on the nCounter Analysis System (NanoString Technologies) according to the manufacturer's protocol. Total RNA was loaded at $100 \mathrm{ng}$ per sample, hybridizations were 17-22 h long, and counts were gathered by scanning on HIGH mode for 280 fields of view per sample. Normalization and analysis of the NanoString panel data were performed using nSolver Software (NanoString Technologies). Base threshold was set to 20 counts. Global count normalization was performed using the geometric mean method as described in the nCounter Expression Data Analysis Guide (NanoString Technologies MAN-C0011-02). Linear ratio of normalized miRNA panel values was calculated by dividing the geometric mean of each experimental group 
by the geometric mean of the control, following the method described in the Gene Expression Data Analysis Guidelines (NanoString Technologies MAN-C0011-04). For each miRNA species, Log2 of each abiraterone and vehicle control value divided by the geometric mean of the vehicle control was calculated. The paired Student's t-test was used to identify significant change in miRNA ratio. The Log2 fold-change values were plotted against the $-\log 10$ of the P-value for each miRNA species to generate the volcano plot.

miRNA qPCR. MiRNA relative expression was quantified with TaqMan Advanced miRNA assays (Thermofisher). Normalized NanoString panel data, containing the top 100 most highly expressed species, was input into RefFinder ${ }^{53}$ to identify relatively highly expressed stable reference miRNA species: hsa-let-7e-5p, hsa-miR32-5p, and hsa-miR-148b-3p. cDNA synthesis was performed using the Taqman Advanced miRNA Synthesis Kit (Thermofisher, Cat\#: A28007) following the manufacturers protocol with a $10 \mathrm{ng}$ total RNA input. qPCR was performed following manufacturer's instructions using TaqMan Fast Advanced MasterMix (Thermofisher Cat\#: 4444556) and Taqman Advanced miRNA Assays: hsa-let-7e-5p (Cat\#: 478579_miR), hsa-miR-32-5p (Cat\#: 478026_miR), hsa-miR-148b-3p (Cat\#: 477824_miR), and hsa-miR-579-3p (Cat\#: 479059_miR). Relative miRNA expression was calculated using the $2^{-\Delta \Delta C t}$ method, comparing hsa-miR-579-3p Ct to the corresponding mean of the stable reference miRNA species.

Construction of SLCO1B3 3'UTR luciferase reporter plasmid and reporter assays. The SLCO1B3 3'UTR was synthesized with flanking NotI and XhoI restriction sites in a pUC57 vector by GENEWIZ, Inc (South Plainfield, NJ). The synthesized sequence was then cloned into complementary restriction sites in a psiCHECK-2 vector, which contains both renilla and firefly luciferase reporter genes (Promega, CAT\#: C8021). The constructed plasmid was sequenced to verify successful cloning.

22Rv1 cells were seeded in a 96-well dish for $24 \mathrm{~h}$ before transfection with $100 \mathrm{ng}$ of psiCHECK-2 empty vector or 3'UTR plasmid using Lipofectamine 2000 (Life Technologies, Cat\#: 11668019). The following day, cells were treated with $20 \mu \mathrm{M}$ abiraterone (SelleckChem, Cat\#: S1123) or vehicle control (0.4\% DMSO) (Sigma Aldrich). After a 24-h treatment period, samples were lysed and assayed using the Dual-Luciferase Reporter Assay System (Promega, Cat\#: E1910) per manufacturer's protocol. Raw luminescence was measured for Renilla and firefly reporters using SpectraMax iD3 (Molecular Devices). For microRNA mimic treated samples, cells were instead co-transfected for $24 \mathrm{~h}$ with the psiCHECK-2 plasmid or empty vector and 1 pmol of hsa-miR-579-3p miRvana mimic or negative control (Life Technologies, Cat\#: 4464066) before performing the reporter assay.

Statistical considerations. The Mann-Whitney U test was performed to test for differences in gene expression when multiple different drug treatments were performed. To assess the significance drug treatment gradients and drug class effects, Dunnett's multiple comparison test was performed. Mann-Whitney U test was used to determine significance of changes in relative expression levels of hsa-miR-579-3p. All comparisons were conducted using GraphPad Prism software (GraphPad Prism version 6.00, GraphPad Software, La Jolla CA).

Received: 2 September 2020; Accepted: 30 April 2021

Published online: 24 May 2021

\section{References}

1. Sissung, T. M. et al. Differential expression of OATP1B3 mediates unconjugated testosterone influx. Mol. Cancer Res. 15, 1096-1105. https://doi.org/10.1158/1541-7786.MCR-16-0477 (2017).

2. Wright, J. L. et al. Expression of SLCO transport genes in castration-resistant prostate cancer and impact of genetic variation in SLCO1B3 and SLCO2B1 on prostate cancer outcomes. Cancer Epidemiol. Biomark. Prev. 20, 619-627. https://doi.org/10.1158/ 1055-9965.EPI-10-1023 (2011).

3. Hamada, A. et al. Effect of SLCO1B3 haplotype on testosterone transport and clinical outcome in Caucasian patients with androgenindependent prostatic cancer. Clin. Cancer Res. 14, 3312-3318. https://doi.org/10.1158/1078-0432.CCR-07-4118 (2008).

4. Sharifi, N. et al. A polymorphism in a transporter of testosterone is a determinant of androgen independence in prostate cancer. BJU Int. 102, 617-621. https://doi.org/10.1111/j.1464-410X.2008.07629.x (2008).

5. Yang, M. et al. SLCO2B1 and SLCO1B3 may determine time to progression for patients receiving androgen deprivation therapy for prostate cancer. J. Clin. Oncol. 29, 2565-2573. https://doi.org/10.1200/JCO.2010.31.2405 (2011).

6. Wood, M. et al. Hormonal regulation of hepatic organic anion transporting polypeptides. Mol. Pharmacol. 68, 218-225. https:// doi.org/10.1124/mol.104.010371 (2005).

7. Jung, D. et al. Characterization of the human OATP-C (SLC21A6) gene promoter and regulation of liver-specific OATP genes by hepatocyte nuclear factor 1 alpha. J. Biol. Chem. 276, 37206-37214. https://doi.org/10.1074/jbc.M103988200 (2001).

8. Vavricka, S. R. et al. The human organic anion transporting polypeptide 8 (SLCO1B3) gene is transcriptionally repressed by hepatocyte nuclear factor 3beta in hepatocellular carcinoma. J. Hepatol. 40, 212-218. https://doi.org/10.1016/j.jhep.2003.10.008 (2004).

9. Jigorel, E., Le Vee, M., Boursier-Neyret, C., Parmentier, Y. \& Fardel, O. Differential regulation of sinusoidal and canalicular hepatic drug transporter expression by xenobiotics activating drug-sensing receptors in primary human hepatocytes. Drug Metab. Dispos. 34, 1756-1763. https://doi.org/10.1124/dmd.106.010033 (2006).

10. Han, S., Kim, K., Thakkar, N., Kim, D. \& Lee, W. Role of hypoxia inducible factor-1alpha in the regulation of the cancer-specific variant of organic anion transporting polypeptide 1B3 (OATP1B3), in colon and pancreatic cancer. Biochem. Pharmacol. 86, 816-823. https://doi.org/10.1016/j.bcp.2013.07.020 (2013).

11. Ramachandran, A. et al. An in vivo hypoxia metagene identifies the novel hypoxia inducible factor target gene SLCO1B3. Eur. J. Cancer 49, 1741-1751. https://doi.org/10.1016/j.ejca.2012.12.003 (2013).

12. Ichihara, S. et al. DNA methylation profiles of organic anion transporting polypeptide 1B3 in cancer cell lines. Pharm. Res. 27, 510-516. https://doi.org/10.1007/s1 1095-010-0064-3 (2010). 
13. Imai, S. et al. Epigenetic regulation of organic anion transporting polypeptide 1B3 in cancer cell lines. Pharm. Res. 30, $2880-2890$. https://doi.org/10.1007/s11095-013-1117-1 (2013).

14. Furihata, T., Sun, Y. \& Chiba, K. Cancer-type organic anion transporting polypeptide 1B3: Current knowledge of the gene structure, expression profile, functional implications and future perspectives. Curr. Drug Metab. 16, 474-485. https://doi.org/10.2174/13892 $00216666150812142715(2015)$.

15. Heemers, H. V. et al. Androgen deprivation increases p300 expression in prostate cancer cells. Cancer Res. 67, 3422-3430. https:// doi.org/10.1158/0008-5472.CAN-06-2836 (2007).

16. Hausser, J. \& Zavolan, M. Identification and consequences of miRNA-target interactions-beyond repression of gene expression. Nat. Rev. Genet. 15, 599-612. https://doi.org/10.1038/nrg3765 (2014).

17. Bruckmueller, H. et al. Clinically relevant multidrug transporters are regulated by microRNAs along the human intestine. Mol. Pharm. 14, 2245-2253. https://doi.org/10.1021/acs.molpharmaceut.7b00076 (2017).

18. Benson, E. A. et al. Rifampin regulation of drug transporters gene expression and the association of microRNAs in human hepatocytes. Front. Pharmacol. 7, 111. https://doi.org/10.3389/fphar.2016.00111 (2016).

19. Krattinger, R. et al. microRNA-192 suppresses the expression of the farnesoid X receptor. Am. J. Physiol. Gastrointest. Liver Physiol. 310, G1044-G1051. https://doi.org/10.1152/ajpgi.00297.2015 (2016).

20. Ganapathy, K. et al. Multifaceted function of microRNA-299-3p fosters an antitumor environment through modulation of androgen receptor and VEGFA signaling pathways in prostate cancer. Sci. Rep. 10, 5167. https://doi.org/10.1038/s41598-020-62038-3 (2020).

21. Kim, J., Wu, L., Zhao, J. C., Jin, H. J. \& Yu, J. TMPRSS2-ERG gene fusions induce prostate tumorigenesis by modulating microRNA miR-200c. Oncogene 33, 5183-5192. https://doi.org/10.1038/onc.2013.461 (2014).

22. Lu, J., Mu, X., Yin, Q. \& Hu, K. miR-106a contributes to prostate carcinoma progression through PTEN. Oncol. Lett. 17, 1327-1332. https://doi.org/10.3892/ol.2018.9697 (2019).

23. Nip, H. et al. Oncogenic microRNA-4534 regulates PTEN pathway in prostate cancer. Oncotarget 7, 68371-68384. https://doi.org/ 10.18632/oncotarget.12031 (2016).

24. Comuzzi, B. et al. The androgen receptor co-activator CBP is up-regulated following androgen withdrawal and is highly expressed in advanced prostate cancer. J. Pathol. 204, 159-166. https://doi.org/10.1002/path.1609 (2004).

25. Antonarakis, E. S. et al. AR-V7 and resistance to enzalutamide and abiraterone in prostate cancer. N. Engl. J. Med. 371, 1028-1038. https://doi.org/10.1056/NEJMoa1315815 (2014).

26. Liu, C., Armstrong, C., Zhu, Y., Lou, W. \& Gao, A. C. Niclosamide enhances abiraterone treatment via inhibition of androgen receptor variants in castration resistant prostate cancer. Oncotarget 7, 32210-32220. https://doi.org/10.18632/oncotarget.8493 (2016).

27. Lombard, A. P. et al. Intra versus inter cross-resistance determines treatment sequence between taxane and AR-targeting therapies in advanced prostate cancer. Mol. Cancer Ther. 17, 2197-2205. https://doi.org/10.1158/1535-7163.MCT-17-1269 (2018).

28. van Bokhoven, A. et al. Molecular characterization of human prostate carcinoma cell lines. Prostate 57, 205-225. https://doi.org/ $10.1002 /$ pros.10290 (2003)

29. Ylitalo, E. B. et al. Marked response to cabazitaxel in prostate cancer xenografts expressing androgen receptor variant 7 and reversion of acquired resistance by anti-androgens. Prostate 80, 214-224. https://doi.org/10.1002/pros.23935 (2020).

30. Agarwal, V., Bell, G. W., Nam, J. W. \& Bartel, D. P. Predicting effective microRNA target sites in mammalian mRNAs. Elife https:// doi.org/10.7554/eLife.05005 (2015).

31. Betel, D., Wilson, M., Gabow, A., Marks, D. S. \& Sander, C. The microRNAorg resource: targets and expression. Nucleic Acids Res. 36, D149-D153. https://doi.org/10.1093/nar/gkm995 (2008).

32. Betel, D., Koppal, A., Agius, P., Sander, C. \& Leslie, C. Comprehensive modeling of microRNA targets predicts functional nonconserved and non-canonical sites. Genome Biol. 11, R90. https://doi.org/10.1186/gb-2010-11-8-r90 (2010).

33. Giatromanolaki, A. et al. CYP17A1 and androgen-receptor expression in prostate carcinoma tissues and cancer cell lines. Curr. Urol. 13, 157-165. https://doi.org/10.1159/000499276 (2019).

34. Xiao, F., Yang, M., Xu, Y. \& Vongsangnak, W. Comparisons of prostate cancer inhibitors abiraterone and TOK-001 binding with CYP17A1 through molecular dynamics. Comput. Struct. Biotechnol. J. 13, 520-527. https://doi.org/10.1016/j.csbj.2015.10.001 (2015).

35. Alex, A. B., Pal, S. K. \& Agarwal, N. CYP17 inhibitors in prostate cancer: Latest evidence and clinical potential. Ther. Adv. Med. Oncol. 8, 267-275. https://doi.org/10.1177/1758834016642370 (2016).

36. Petrunak, E. M., Rogers, S. A., Aube, J. \& Scott, E. E. Structural and functional evaluation of clinically relevant inhibitors of steroidogenic cytochrome P450 17A1. Drug Metab. Dispos. 45, 635-645. https://doi.org/10.1124/dmd.117.075317 (2017).

37. Wu, R. R., Zhong, Q., Liu, H. F. \& Liu, S. B. Role of miR-579-3p in the development of squamous cell lung carcinoma and the regulatory mechanisms. Eur. Rev. Med. Pharmacol.Sci. 23, 9464-9470. https://doi.org/10.26355/eurrev_201911_19440 (2019).

38. Angius, A. et al. MicroRNA-425-5p expression affects BRAF/RAS/MAPK pathways in colorectal cancers. Int. J. Med. Sci. 16, 1480-1491. https://doi.org/10.7150/ijms.35269 (2019).

39. Kalhori, M. R., Irani, S., Soleimani, M., Arefian, E. \& Kouhkan, F. The effect of miR-579 on the PI3K/AKT pathway in human glioblastoma PTEN mutant cell lines. J. Cell Biochem. 120, 16760-16774. https://doi.org/10.1002/jcb.28935 (2019).

40. Wang, X. et al. Targeting signal-transducer-and-activator-of-transcription 3 sensitizes human cutaneous melanoma cells to BRAF inhibitor. Cancer Biomark. 23, 67-77. https://doi.org/10.3233/CBM-181365 (2018).

41. Ohtsuka, H. et al. Farnesoid X receptor, hepatocyte nuclear factors 1alpha and 3beta are essential for transcriptional activation of the liver-specific organic anion transporter-2 gene. J. Gastroenterol. 41, 369-377. https://doi.org/10.1007/s00535-006-1784-3 (2006).

42. Zedan, A. H., Osther, P. J. S., Assenholt, J., Madsen, J. S. \& Hansen, T. F. Circulating miR-141 and miR-375 are associated with treatment outcome in metastatic castration resistant prostate cancer. Sci. Rep. 10, 227. https://doi.org/10.1038/s41598-019-57101-7 (2020).

43. Mostaghel, E. A. et al. Association of tissue abiraterone levels and SLCO genotype with intraprostatic steroids and pathologic response in men with high-risk localized prostate cancer. Clin. Cancer Res. 23, 4592-4601. https://doi.org/10.1158/1078-0432. CCR-16-2245 (2017)

44. Fredsoe, J. et al. Profiling of circulating microRNAs in prostate cancer reveals diagnostic biomarker potential. Diagnostics (Basel). https://doi.org/10.3390/diagnostics10040188 (2020).

45. Wang, F. et al. microRNA-16-5p enhances radiosensitivity through modulating Cyclin D1/E1-pRb-E2F1 pathway in prostate cancer cells. J. Cell Physiol. 234, 13182-13190. https://doi.org/10.1002/jcp.27989 (2019).

46. Akbayir, S., Muslu, N., Erden, S. \& Bozlu, M. Diagnostic value of microRNAs in prostate cancer patients with prostate specific antigen (PSA) levels between 2, and $10 \mathrm{ng} / \mathrm{mL}$. Turk. J. Urol. 42, 247-255. https://doi.org/10.5152/tud.2016.52463 (2016).

47. Shen, H. et al. miR-181a-5p is downregulated and inhibits proliferation and the cell cycle in prostate cancer. Int. J. Clin. Exp. Pathol. 11,3969-3976 (2018).

48. Zhiping, C. et al. MiR-181 a promotes epithelial to mesenchymal transition of prostate cancer cells by targeting TGIF2. Eur. Rev. Med. Pharmacol. Sci. 21, 4835-4843 (2017).

49. Li, Y. et al. miR-181a-5p inhibits cancer cell migration and angiogenesis via downregulation of matrix metalloproteinase-14. Cancer Res. 75, 2674-2685. https://doi.org/10.1158/0008-5472.CAN-14-2875 (2015). 
50. Su, S. F. et al. miR-30d, miR-181a and miR-199a-5p cooperatively suppress the endoplasmic reticulum chaperone and signaling regulator GRP78 in cancer. Oncogene 32, 4694-4701. https://doi.org/10.1038/onc.2012.483 (2013).

51. Liao, Y. et al. Targeting GRP78-dependent AR-V7 protein degradation overcomes castration-resistance in prostate cancer therapy. Theranostics 10, 3366-3381. https://doi.org/10.7150/thno.41849 (2020).

52. Li, Z. et al. Conversion of abiraterone to D4A drives anti-tumour activity in prostate cancer. Nature 523, 347-351. https://doi.org/ 10.1038/nature14406 (2015).

53. Xie, F., Xiao, P., Chen, D., Xu, L. \& Zhang, B. miRDeepFinder: A miRNA analysis tool for deep sequencing of plant small RNAs. Plant Mol. Biol. https://doi.org/10.1007/s11103-012-9885-2 (2012).

\section{Acknowledgements}

The authors would like to thank Dr. Tristan M. Sissung for his helpful comments and suggestions. The content of this publication does not necessarily reflect the views or policies of the Department of Health and Human Services, nor does mention of trade names, commercial products, or organization imply endorsement by the U.S. Government.

\section{Author contributions}

D.K.P., C.H.C, and W.D.F. designed and supervised the study and provided research materials and resources. R.H.B., E.M.M., J.D.S., K.Y.L., E.N.R, C.H.C. developed methodologies. R.H.B., E.M.M., J.D.S., K.Y.L., E.N.R. conducted experiments and validated results. R.H.B., E.M.M., K.Y.L., C.H.C. performed data analysis. R.H.B., E.M.M., and C.H.C. wrote the manuscript. All authors reviewed, edited, and approved the final manuscript.

\section{Funding}

This study was supported by the Intramural Research Program of the National Institutes of Health, National Cancer Institute, Bethesda, MD, US (ZIA BC 010453).

\section{Competing interests}

The authors declare no competing interests.

\section{Additional information}

Supplementary Information The online version contains supplementary material available at https://doi.org/ 10.1038/s41598-021-90143-4.

Correspondence and requests for materials should be addressed to W.D.F.

Reprints and permissions information is available at www.nature.com/reprints.

Publisher's note Springer Nature remains neutral with regard to jurisdictional claims in published maps and institutional affiliations.

(c) Open Access This article is licensed under a Creative Commons Attribution 4.0 International License, which permits use, sharing, adaptation, distribution and reproduction in any medium or format, as long as you give appropriate credit to the original author(s) and the source, provide a link to the Creative Commons licence, and indicate if changes were made. The images or other third party material in this article are included in the article's Creative Commons licence, unless indicated otherwise in a credit line to the material. If material is not included in the article's Creative Commons licence and your intended use is not permitted by statutory regulation or exceeds the permitted use, you will need to obtain permission directly from the copyright holder. To view a copy of this licence, visit http://creativecommons.org/licenses/by/4.0/.

This is a U.S. Government work and not under copyright protection in the US; foreign copyright protection may apply 2021 